01.1;02.3

\title{
Работа образования кластера новой фазы, который находится в однородном внешнем электрическом поле и поле иона вне кластера
}

\author{
(C) В.А. Власов
}

Институт криосферы Земли Тюменского научного центра СО РАН, Тюмень, Россия

E-mail: vlasov.ikz@gmail.com

Поступило в Редакцию 7 декабря 2021 г.

В окончательной редакции 28 декабря 2021 г.

Принято к публикации 13 января 2022 г.

В рамках классического термодинамического подхода получено выражение для совместного вклада в работу образования сферического кластера новой фазы со стороны однородного внешнего электрического поля и поля иона, который находится вне кластера. Проведен анализ полученного выражения в случае фазового перехода пар-жидкость.

Ключевые слова: нуклеация, работа образования кластера, электрическое поле, ионы, скорость нуклеации.

DOI: 10.21883/PJTF.2022.06.52213.19100

Наличие электрического поля является фактором, влияющим на устойчивость метастабильных фаз и скорость нуклеации $[1,2]$. Поэтому контролируемая нуклеация в электрических полях находит применение в различных технологиях, например в технологии получения наноразмерных частиц [3].

При вычислении работы образования кластера новой фазы в электрическом поле, как правило, рассматривают только два случая. В первом из них считается, что кластер образуется в поле иона $[4,5]$. Во втором случае считается, что кластер образуется в однородном внешнем поле [6,7]. Однако в природе и технике часто встречаются ситуации, когда при образовании кластера новой фазы ионы и однородное внешнее электрическое поле присутствуют одновременно. В [8] получено выражение для работы образования кластера новой фазы в случае, когда кластер образуется на ионе при наличии однородного внешнего поля. В настоящей работе рассмотрен случай образования кластера новой фазы, который одновременно находится в однородном внешнем поле и поле иона вне кластера. Данный случай важен, поскольку в процессе образования кластера может происходить вытеснение иона на периферию этого кластера $[9,10]$.

Основная задача при вычислении работы образования кластера новой фазы в электрическом поле состоит в вычислении вклада в эту работу со стороны электрического поля. Можно показать $[11,12]$, что общее выражение для вклада в работу образования кластера новой фазы со стороны электрического поля имеет следующий вид:

$$
W_{e l}=\frac{\varepsilon_{0}}{2}\left(\varepsilon_{\text {old }}-\varepsilon_{\text {new }}\right) \int_{V_{c l}} \nabla \varphi \cdot \nabla \tilde{\varphi} d V,
$$

где $\varepsilon_{0}-$ электрическая постоянная, $\varepsilon_{\text {old }}$ и $\varepsilon_{\text {new }}-$ статическая диэлектрическая проницаемость старой и новой фазы соответственно, $\varphi$ и $\tilde{\varphi}$ - электростатический потенциал в отсутствие и в присутствии кластера соответственно. В выражении (1) объемное интегрирование ведется по объему кластера $V_{c l}$. Далее определим величину $W_{e l}$ для рассматриваемого в настоящей работе случая, предполагая для простоты, что кластер новой фазы имеет сферическую форму.

Будем использовать сферическую систему координат, выбрав ее начало в центре сферического кластера (рис. 1). При этом ось $z$, от которой отчитывается полярный угол $\theta$, направим вдоль напряженности однородного электрического поля в старой фазе $\mathbf{E}$.

В силу принципа суперпозиции потенциал $\varphi$ можно представить в виде

$$
\varphi=\varphi_{\text {field }}+\varphi_{\text {ion }},
$$

где $\varphi_{\text {field }}$ - электростатический потенциал однородного поля в отсутствие кластера, $\varphi_{i o n}$ - электростатический потенциал поля иона в отсутствие кластера. Потенциал $\varphi_{\text {field }}$ в выбранной системе координат задается как

$$
\varphi_{\text {field }}=-E r \cos \theta
$$

а потенциал $\varphi_{i o n}$ вне иона задается как

$$
\begin{aligned}
\varphi_{\text {ion }} & =\frac{q}{4 \pi \varepsilon_{0} \varepsilon_{\text {old }} r_{p}}=\frac{q}{\varepsilon_{0} \varepsilon_{\text {old }} r_{0}} \sum_{l=0}^{\infty} \sum_{m=-l}^{l} \frac{1}{2 l+1}\left(\frac{r}{r_{0}}\right)^{l} \\
& \times Y_{l m}^{*}\left(\Omega_{0}\right) Y_{l m}(\Omega), \quad r<r_{0} .
\end{aligned}
$$

Здесь $r$ - расстояние от центра кластера до точки наблюдения $P ; q-$ электрический заряд иона; $r_{p}-$ расстояние от центра иона до точки наблюдения $P ; r_{0}-$ расстояние от центра кластера до центра иона; $Y_{l m}(\Omega)-$ сферические функции; $\Omega \equiv\{\theta, \psi\}$ - набор сферических углов радиус-вектора, проведенного от центра кластера 


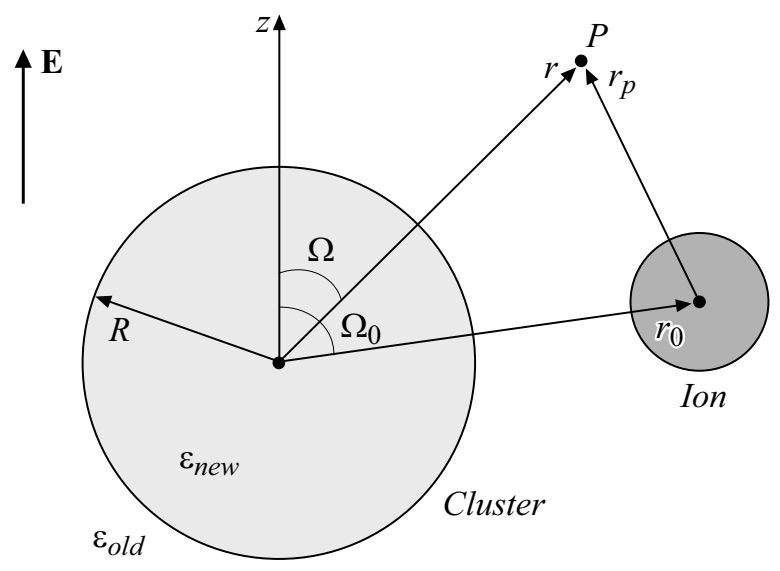

Рис. 1. Геометрия задачи.

до точки наблюдения $P(\psi-$ азимутальный угол); $\Omega_{0} \equiv\left\{\theta_{0}, \psi_{0}\right\}-$ набор сферических углов радиусвектора, проведенного от центра кластера до центра иона. После подстановки выражений (3) и (4) в (2) получим окончательное выражение для потенциала $\varphi$.

Принцип суперпозиции позволяет представить потенциал $\tilde{\varphi}$ как

$$
\tilde{\varphi}=\tilde{\varphi}_{\text {field }}+\tilde{\varphi}_{\text {ion }},
$$

где $\tilde{\varphi}_{\text {field }}$ - электростатический потенциал однородного поля в присутствии кластера, $\tilde{\varphi}_{i o n}-$ электростатический потенциал иона в присутствии кластера. Можно показать [13], что

$$
\tilde{\varphi}_{\text {field }}=-\frac{3 \varepsilon_{\text {old }}}{2 \varepsilon_{\text {old }}+\varepsilon_{\text {new }}} E r \cos \theta, \quad r \leqslant R,
$$

где $R$ - радиус кластера. Кроме того, можно показать [14], что

$$
\begin{aligned}
\tilde{\varphi}_{\text {ion }} & =\frac{q}{\varepsilon_{0} r_{0}} \sum_{l=0}^{\infty} \sum_{m=-l}^{l} \frac{1}{\varepsilon_{\text {old }}(l+1)+\varepsilon_{\text {new }} l}\left(\frac{r}{r_{0}}\right)^{l} \\
& \times Y_{l m}^{*}\left(\Omega_{0}\right) Y_{l m}(\Omega), \quad r \leqslant R .
\end{aligned}
$$

После подстановки выражений (6) и (7) в (5) получим окончательное выражение для потенциала $\tilde{\varphi}$ внутри кластера.

Учитывая найденные потенциалы $\varphi$ и $\tilde{\varphi}$, вычислим интеграл в выражении (1). При вычислении этого интеграла учтем, что [15]:

$$
\begin{gathered}
r \cos \theta=\sqrt{\frac{4 \pi}{3}} \sum_{l=0}^{\infty} \sum_{m=-l}^{l} r^{l} Y_{l m}(\Omega) \delta_{l 1} \delta_{m 0}, \\
\nabla r^{l} Y_{l m}(\Omega)=l r^{l-1} \mathbf{Y}_{l m}^{(-1)}(\Omega)+\sqrt{l(l+1)} r^{l-1} \mathbf{Y}_{l m}^{(1)}(\Omega),
\end{gathered}
$$

где $\delta_{i j}-$ символ Кронекера, $\mathbf{Y}_{l m}^{(-1)}(\Omega)$ и $\mathbf{Y}_{l m}^{(1)}(\Omega)-$ шаровые векторы. Кроме того, при вычислении этого интеграла учтем свойства сферических функций и шаровых векторов [15]. В результате получим следующее выражение:

$$
\begin{aligned}
& W_{\text {el }}=\frac{\left(\varepsilon_{\text {old }}-\varepsilon_{\text {new }}\right) q^{2}}{8 \pi \varepsilon_{0} \varepsilon_{\text {old }} R} \sum_{l=0}^{\infty} \frac{l}{\varepsilon_{\text {old }}(l+1)+\varepsilon_{\text {new }} l}\left(\frac{R}{r_{0}}\right)^{2 l+2} \\
& +\frac{2 \pi \varepsilon_{0} \varepsilon_{\text {old }}\left(\varepsilon_{\text {old }}-\varepsilon_{\text {new }}\right) R^{3} E^{2}}{2 \varepsilon_{\text {old }}+\varepsilon_{\text {new }}}-\frac{\left(\varepsilon_{\text {old }}-\varepsilon_{\text {new }}\right) q R^{3} E \cos \theta_{0}}{\left(2 \varepsilon_{\text {old }}+\varepsilon_{\text {new }}\right) r_{0}^{2}} .
\end{aligned}
$$

В отсутствие однородного внешнего электрического поля $(E=0)$ выражение (8) переходит в известное выражение для вклада в работу образования сферического кластера новой фазы со стороны поля иона, находящегося вне кластера [5]. В отсутствие иона $(q=0)$ выражение (8) переходит в известное выражение для вклада в работу образования сферического кластера новой фазы со стороны однородного внешнего электрического поля [6]. Одновременное наличие иона и однородного внешнего электрического поля проявляется в том, что в выражении (8) следует учитывать перекрестное слагаемое, которое зависит от угла $\theta_{0}$.

На рис. 2 в качестве примера приведены расчетные зависимости работы образования кластера новой фазы $W$ от радиуса этого кластера $R$ для полярного вещества метанола в случае фазового перехода пар-жидкость. Данные расчетные зависимости были получены с помощью выражения

$$
W=4 \pi \sigma R^{2}-\frac{4 \pi k T \ln S}{3 v} R^{3}+W_{e l},
$$

где величина $W_{e l}$ задается выражением (8). Здесь $\sigma-$ коэффициент поверхностного натяжения между жидкостью и паром, $k$ - постоянная Больцмана, $T$ - температура, $S$ - пересыщение пара, $v$ - объем, приходящийся на одну молекулу в жидкости.

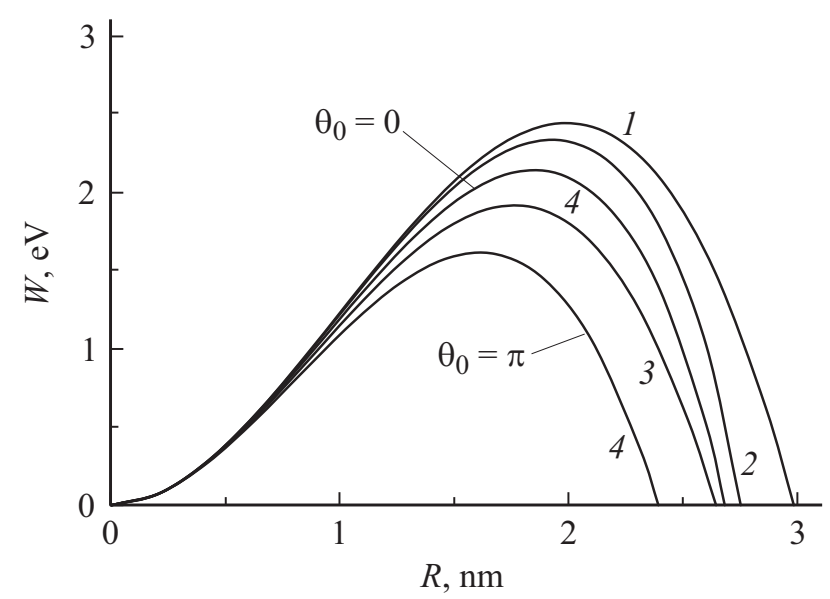

Рис. 2. Зависимости $W$ от $R$ для метанола в случае фазового перехода пар-жидкость при $T=280 \mathrm{~K}, S=1.5$ и $r_{0}=3 \mathrm{~nm}$. $1-q=0$ и $E=0$ (гомогенная нуклеация), $2-q=e(e-$ элементарный электрический заряд) и $E=0,3-q=0$ и $E=500 \mathrm{MV} / \mathrm{m}, 4-q=e$ и $E=500 \mathrm{MV} / \mathrm{m}$. 
Из рис. 2 видно, что в случае фазового перехода пар-жидкость наличие электрического поля приводит к уменьшению критического радиуса кластера новой фазы $R^{*}$, который определяется из условия $d W\left(R^{*}\right) / d R=0$, и к уменьшению работы образования кластера новой фазы критического размера $W^{*}=W\left(R^{*}\right)$. Это в свою очередь приводит к увеличению вероятности появления такого кластера, а значит, и к увеличению скорости нуклеации $J \propto \exp \left(-W^{*} / k T\right)$.

Анализ выражения (9) показывает, что в случае фазового перехода пар-жидкость при наличии однородного внешнего электрического поля и иона, находящегося вне кластера, работа $W^{*}$ существенным образом зависит от угла $\theta_{0}$ при фиксированном расстоянии $r_{0}$. Зависимость от угла $\theta_{0}$ проявляется в том, что в однородном внешнем электрическом поле для положительно заряженного иона работа $W^{*}$ будет максимальной при $\theta_{0}=0$ и минимальной при $\theta_{0}=\pi$ (см. кривые 4 на рис. 2 ). При этом для отрицательно заряженного иона работа $W^{*}$ будет максимальной при $\theta_{0}=\pi$ и минимальной при $\theta_{0}=0$. Таким образом, в случае положительно заряженного иона вероятность образования кластера новой фазы критического размера будет максимальной при $\theta_{0}=\pi$, а в случае отрицательно заряженного иона - при $\theta_{0}=0$.

\section{Финансирование работы}

Работа выполнена по госзаданиям № 121041600040-3 и 1021110317891-3-2.4.2 при финансовой поддержке Российского фонда фундаментальных исследований и Тюменской области (проект № 20-43-720002).

\section{Конфликт интересов}

Автор заявляет, что у него нет конфликта интересов.

\section{Список литературы}

[1] В.С. Воробьев, С.П. Малышенко, ТВТ, 48 (6), 1005 (2010). [V.S. Vorob'ev, S.P. Malyshenko, High Temp., 48 (6), 957 (2010). DOI: 10.1134/S0018151X10060246].

[2] L.F. Alexander, N. Radacsi, CrystEngComm, 21 (34), 5014 (2019). DOI: $10.1039 / \mathrm{c} 9 \mathrm{ce} 00755 \mathrm{e}$

[3] M. Adachi, M. Kusumi, M. Furuta, S. Tsukui, Adv. Powder Technol., 16 (5), 549 (2005). DOI: $10.1163 / 1568552054969924$

[4] М. Фольмер, Кинетика образования новой фбазы (Наука, М., 1986), с. 137-143. [M. Volmer, Kinetik der Phasenbildung (Steinkopff, Dresden, 1939).].

[5] Г.В. Аникин, С.Н. Плотников, ЖФХ, 79 (2), 363 (2005). [G.V. Anikin, S.N. Plotnikov, Russ. J. Phys. Chem., 79 (2), 294 (2005).].

[6] J.O. Isard, Phil. Mag., 35 (3), 817 (1977). DOI: $10.1080 / 14786437708236010$

[7] V.B. Warshavsky, A.K. Shchekin, Coll. Surf. A, 148 (3), 283 (1999). DOI: 10.1016/S0927-7757(98)00769-9
[8] A.K. Shchekin, M.S. Kshevetskiy, V.B. Warshavsky, Coll. Surf. A, 223 (1-3), 277 (2003). DOI: $10.1016 / \mathrm{S} 0927-7757(03) 00201-2$

[9] С.В. Шевкунов, ТВТ, 50 (2), 274 (2012). [S.V. Shevkunov, High Temp., 50 (2), 255 (2012). DOI: $10.1134 / \mathrm{S} 0018151 \mathrm{X} 12020149]$.

[10] M. Zhang, F. Han, C. Li, P. Wang, Y. Yang, K. Yu, Chem. Phys., 539, 110932 (2020). DOI: $10.1016 /$ j.chemphys.2020.110932

[11] Л.Д. Ландау, Е.М. Лифшиц, Электродинамика сплошных сред (Наука, М., 1982), с. 75-79.

[12] D. Kashchiev, Nucleation: basic theory with applications (Butterworth-Heinemann, Oxford, 2000), p. 315-316.

[13] А.Н. Тихонов, А.А. Самарский, Уравнения математической физики (Изд-во МГУ, М., 1999), с. 734-737.

[14] Г.А. Гринберг, Избранные вопросы математической теории электрических и магнитных явлений (Изд-во АН СССР, Л., 1948), с. 188-192.

[15] Д.А. Варшалович, А.Н. Москалев, В.К. Херсонский, Квантовая теория углового момента (Наука, Л., 1975), c. $115-148,183-199$. 